\title{
Enfermedades raras
}

\section{Rare diseases}

"Ninguna enfermedad es tan rara como para no merecer nuestra atención"

Cada día aparecen más y mejores herramientas para el diagnóstico y tratamiento adecuados de las enfermedades que aquejan a la población mundial. El siglo pasado y los inicios de éste han sido testigos de estos acontecimientos. Los grandes descubrimientos muestran que vamos por el camino correcto; tal es el caso del estudio e inicio de la decodificación del genoma humano, que es de vital importancia en el estudio de las enfermedades heredodegenerativas, especialmente las de tipo metabólico y lisosomal etiquetadas como "raras", intratables e incurables en su gran mayoría. Sin embargo, nada más lejos de la realidad, la gran mayoría ahora son previsibles y muchas de ellas tratables.

El propósito de dar a conocer lo anterior es percibir el impacto que estas enfermedades ejercen en los pacientes, sus familiares y en los servicios de salud. De estas enfermedades $80 \%$ tienen origen genético y también en $80 \%$ de los casos se ha detectado el gen causante de la enfermedad; otras son cánceres poco frecuentes, enfermedades autoinmunitarias, malformaciones congénitas o infecciosas.

Hay tres tipos de enfermedades genéticas:

1. Defectos monogénicos: la afección es de un solo gen.
2. Trastornos cromosómicos donde éstos (o parte de ellos) faltan o cambian.

3. Multifactoriales, en los cuales hay mutación en dos o más genes (aquí influyen el ambiente y el estilo de vida).

Se puede heredar una mutación genética de uno o ambos padres, pero también pueden suceder estos cambios durante la vida de un individuo.

El concepto de enfermedades "raras" se acuñó por primera vez a mediados de la década de los años 80 del siglo pasado, en Estados Unidos, relacionado con el concepto de medicamentos "huérfanos". Se denominaron enfermedades "raras" porque se caracterizaban por su baja prevalencia (número de personas viviendo con una enfermedad en un momento dado) e incidencia (número de nuevos diagnósticos en un año). No obstante, tienen altas tasas de mortalidad con evolución crónica muy severa y múltiples deficiencias motoras, sensoriales y cognitivas. Aproximadamente $50 \%$ aparece en la edad pediátrica pero la mayor prevalencia es en adultos debido a la excesiva mortalidad de algunas enfermedades infantiles: $30 \%$ de los niños con este tipo de enfermedades fallece antes de los 5 años y en 35\% de los casos son responsables de las muertes antes del año de edad.

Se conoce como enfermedad "huérfana o rara" cualquier enfermedad que afecte a un pequeño porcentaje de la población, aquella que no 
cuente con tratamientos adecuados o cuando la severidad de la enfermedad sea extrema. Se les denomina así por la amplia diversidad de desórdenes y síntomas no sólo según la enfermedad sino también de pacientes que la padecen. Existen síntomas relativamente comunes que pueden ocultarla y conducir a diagnósticos erróneos.

Se considera entonces que una enfermedad es "rara" cuando afecta, desde el punto de vista poblacional, a menos de cinco personas de cada 10000 habitantes o a uno de cada 5000 nacidos vivos. Sin embargo, hay que tomar en cuenta que este estatus puede variar con el tiempo y también con el área geográfica de donde se obtengan los datos. Por ejemplo, una enfermedad genética o infecciosa puede ser rara en una región pero frecuente en otra, como en el caso de la lepra que es rara en Francia pero muy común en África Central, o el ébola que es endémico en ese mismo continente pero prácticamente desconocido en otras regiones.

Si hablamos de origen genético la talasemia (una anemia hemolítica) es rara en el norte de Europa pero frecuente en la región del mediterráneo, África, Estados Unidos y parte de Sudamérica. Algunas enfermedades infecciosas son frecuentes en países del Tercer Mundo y de baja frecuencia en el Mundo Desarrollado. A éstas se les Ilama también "enfermedades olvidadas" y son así desde el punto de vista geográfico, social y económico, con muy poco apoyo en su inversión e interés para la investigación en su diagnóstico y tratamiento oportunos, lo que conlleva a un círculo vicioso.

Hasta la fecha se tiene registro de entre 6 y 7 mil enfermedades raras; se cree que afectan aproximadamente a una de cada 10 personas, aunque las más frecuentes de éstas son 231 y sólo 70 tienen tratamiento; incluso se sugiere que cada semana se describen 5 nuevas enfermedades "raras" en la literatura médica aunque no existe un criterio científico estandarizado para su verdadera clasificación.

Demográficamente cada país utiliza diferentes fórmulas para definirlas. En Estados Unidos una enfermedad se considera "rara" cuando la padecen menos de 200000 personas; según el acta de enfermedades raras existen cerca de 25 millones de estadounidenses que padecen alguna. En Japón se definen con menos de 4 casos por cada 10000 habitantes o aquellas que afectan a menos de 50000 personas. En Europa la Comisión Europea de Salud establece que una enfermedad se califica como "rara" cuando afecta a 1 de 50000 personas y se estima que el número de personas que las padecen es superior a los 30 millones; estos pacientes representan de 6 a $8 \%$ de la población de la Unión Europea y existen en su territorio de 5 mil a 7 mil diferentes tipos de estas enfermedades.

En Iberoamérica hay aproximadamente $42 \mathrm{mi}$ Ilones de personas con enfermedades raras. En nuestro medio $7 \%$ de la población mexicana padece alguna de estas enfermedades y se estima que hay alrededor de 6 millones de personas que las padecen.

El proyecto genes globales informa que alrededor de 350 millones de personas en todo el mundo están afectadas por alguna enfermedad "rara" (aproximadamente 7\%) y que su impacto mundial es mayor que los del cáncer y el sida juntos.

Estas son las enfermedades más "raras" y curiosas del mundo:

1. Sinestesia: estimulación simultánea y múltiple de los sentidos.

2. Síndrome del acento extranjero: hablar en lengua materna con acento extranjero.

3. Síndrome de Capgras: la persona cree que sus familiares son impostores. 
4. Síndrome de Cotard: pacientes con delirio de negación (el paciente cree haber fallecido).

5. Micropsia o macropsia: "Síndrome de Alicia en el país de las maravillas" (percepción de que las cosas son demasiado pequeñas o grandes).

6. Síndrome Riley-Day: los pacientes no perciben el dolor.

7. Síndrome de Proteus: crecimiento exagerado de la piel y huesos: "el hombre elefante".

8. Síndrome de Möbius: inexpresión facial y ocular por alteración del sexto y séptimo pares craneales.

9. Progeria de Hutchinson-Gilfors: envejecimiento prematuro.

10. Síndrome de apnea-hipopnea durante el sueño: no existen las señales nerviosas necesarias para que se dé la respiración en el sueño.

11. Deficiencia de ribosa-5-fosfato isomerasa: sólo se ha descrito en un paciente hasta la fecha.

Las pruebas genéticas son exámenes de sangre y de otros tejidos para detectar dichos trastornos y existen más de 200 disponibles que se relizan por varios motivos:

1. Encontrar trastornos genéticos en el feto.

2. Saber si las personas tienen un gen de la enfermedad y pueden transferírsela a sus hijos.

3. Estudiar embriones para detectar enfermedades.

4. Evaluar la presencia de enfermedades genéticas en adultos antes de que se produzcan síntomas.

5. Hacer el diagnóstico en una persona con síntomas de una enfermedad rara.
6. Determinar el tipo o dosis de medicamento que es mejor para la persona.

Los resultados de las pruebas pueden ayudar a que una persona tome decisiones como la planificación familiar o la cobertura de un seguro.

Se debe dar asesoramiento genético a la mujer cuando:

1. Tiene o le preocupa tener una enfermedad hereditaria.

2. Está embarazada o planea estarlo después de los 35 años de edad.

3. Ya tiene un hijo con un trastorno o defecto congénito.

4. Tuvo dos o más pérdidas de embarazo o un niño que falleció.

5. Se realizó un ultrasonido o pruebas que sugieren la posibilidad de un problema.

El promedio de tiempo para llegar a un diagnóstico final puede variar de 5 a 10 años (30\%) y requerir revisiones de más de 10 médicos (20\%). Las enfermedades "raras" no sólo afectan a las personas diagnosticadas, sino a sus familias, amigos, cuidadores y a toda la sociedad. La mayoría tiene curso clínico crónico debilitante con esperanza de vida reducida. Coexisten la denominada pluridiscapacidad (80\%) con un alto grado de dependencia y mortalidad de $50 \%$. La discapacidad produce aislamiento y discriminación, reduce las oportunidades educativas, profesionales y sociales y con ello conduce a deficiencias físicas, psicológicas e intelectuales.

En cuanto al impacto económico se estima que las familias de estos enfermos destinan más de $50 \%$ de sus ingresos para su cuidado. Existen organizaciones de pacientes para aumentar el conocimiento, diagnóstico oportuno y tratamiento de estas enfermedades, como La Organización 
Nacional para Enfermedades Raras que fue creada en 1983 por individuos que las padecen y por sus familias.

En el año 2002 se creó la Oficina de Investigación de Enfermedades Raras, en el Instituto Nacional de Salud de Estados Unidos, que se encarga de realizar investigaciones clínicas y biomédicas para el desarrollo de terapias para este tipo de enfermedades. Ese mismo año se firmó el acta de "Desarrollo de Productos Huérfanos para el tratamiento de Enfermedades Raras" y se propusieron iniciativas similares en Europa.

El primer Día de las Enfermedades Raras fue organizado por Europa y Canadá, al mismo tiempo, en 2008. Ese día fue creado para aumentar la concientización acerca de las enfermedades raras y se conmemora el último día de febrero de cada año. A partir de entonces se han creado diversas organizaciones no gubernamentales de apoyo a lo largo de todo el mundo; existen hoy en día más de 500 .

En México, en abril del 2011, se creó la Federación Mexicana para Enfermedades Raras y la Asociación Mexicana de Enfermedades Lisosomales en 2013.

\section{LECTURAS RECOMENDADAS}

1. International Conference on Rare Diseases and Orphan Drugs (ICORD) «The Yukiwariso Declaration» (http://icord. se/main-menu/yukiwariso).

2. Prevalence of rare diseases: Bibliographic data. Listed in order of decreasingprevalence or number of published case. Orphanet Report Series, Rare Diseases collection. Nov. 2013. http://www.orpha.net/orphacom/cahiers/ docs/GB/Prevalence of_rare_diseases_by_decreasingprevalence_or_cases.pdf).

3. OMS (2012). Unidos para combatir las enfermedades raras. Boletín de la Organización Mundial de la Salud 2012;90(6):471-476. http://www.who. int/bulletin/volumes/90/6/12-020612/

4. Useful Information on Rare Diseases from an EU Perspective. European Commission. http://ec.europa.eu/health/ ph_information/documents/ev20040705_rd05_en.pdf
5. Comisión Europea. Las enfermedades raras: un desafío para Europa. http://ec.europa.eu/health/ph_threats/non_com/ docs/raredis_com_es.pdt

6. Public Law 97-414: Orphan Drug Act (1983), designation of drugs for' rare diseases or conditions, en www.fda.gov http://www.fda.gov/regulatoryinformation/legislation/ federalfooddrugandcosmeticactfdcact/significantamendmentothefcact/orphandrugact/default.htm

7. Rare Diseases: Understanding This Public Health Priority (http://www.eurordis.org/IMG/pdf/princeps_documentEN.pdf). European Organization for Rare Diseases (EURORDIS). Nov. 2005

8. February 29 is Rare Disease Day in Europe. http://www. novartis.com/newsroom/news/2008-02-28_rare-diseaseday.shtml

9. Rare Disease en: http://ec.europa.eu/health-eu/health problems/rare_diseases/index_en.htm

10. van de Laar FA, Bor $\mathrm{H}$, van de Lisdonk EH.Prevalence of zebras in general practice: data from the Continuous Morbidity Registration Nijmegen. Eur J Gen Pract. 14. 2008;Suppl1(s1):44-6. doi:10.1080/13814780802436176

11. What is a Genetic Disease. http://www.geneticalliance.org/ ws_display.asp?filter=diseases\&char $=\% 23 \&$ s_Diseases= Genetic Alliance

12. "The National Organization for Rare Disorders (NORD) (http:// www.reuters.com/article/pressRelease/idUS81376+05May-2008+BW20080505) Reuters. 5 de mayo de 2008.

13. Canadian Organization for Rare Disorders. (http://www. raredisorders.ca).

14. Federación Española de Enfermedades Raras (FEDER) Quienes somos (httpl/www.enfermedades-raras.org/ index.php?option=com_content\&view=article \&id=289\&l temid=130)

15. Fundación Geiser-Misión, Visión, objetivos. http://www. fundaciongeiser.orglgeiserlmision-vision-objetivos/

16. Federación Colombiana de Enfermedades Raras (FECOER) - Nuestra Historia. http://www.fecoer.org/nuestra-historia/

17. Decision No 1295/1999/EC of the European Parliament and of the Council of 29 April 1999 adopting a programme of Community action on rare diseases within the framework for action in the field of public health (1999 to 2003): http:/europa.eu.int/eur-lex/pri/en/oj/dat/1999/ I_155/1_15519990622en00010005.pdf

18. Consulta Pública las Enfermedades Raras: Un Desafío para Europa. Comisión Europea Dirección General de Sanidad y Protección de los Consumidores Comisión Europea, L-2920 Luxemburgo. 2008 http://ec.europa.eu/health/ ph_threats/non_com/docs/raredis_comm_es.pdf

19. Rmialle-gómara E, González MA, Perucha M, Quiñones C, Lezaun ME, Posada de la Paz M, en nombre del Grupo REpIER (Red de Investigación Epidemiológica de Enfermedades Raras). Mortalidad por la enfermedad de Huntington en España en el período 1981- 2004. Rev Neurol 2007; 45:88-90. 
20. Botella Rocamora P, Zurriaga Lloréns O, Posada de la Paz M, Martínez Beneito MA, Belprieto E, Robustillo Rodela A. en el nombre de REpIER. Atlas Nacional/Provincial de Enfermedades Raras 1999-2003. REpIER editores. 2006.

21. Zurriaga Lloréns $O$, Martínez García C, Arizo Luque V, Sánchez Pérez MJ, Ramos Aceitero JM, García Blasco MJ en nombre de los integrantes de la Red REpIER. Los Registros de Enfermedades en la Investigación Epidemiológica de las Enfermedades Raras en España. Rev Esp Salud Pública 2005;80:249-257.

22. Sánchez-Valle $E$, Posada $M$, Villaverde-Hueso $A$, Touriño E, Ferrari-Arroyo MJ, Boada L. Estimating the burden of disease for autism spectrum disorders in Spain in 2003. J Autism Dev Disord DOI 10.1007/s10803- 007-0393-1.

23. The genetic conception of health: is it as radical as claimed? Petersen A. Health 2006;10:481-500.

24. World Health Organization. International Statistical Classification of Diseases and Related Health Problems 10th Revision. Version for 2006. http:/jwww.who.int/classifications/apps/icd/icdlOonline/

25. Orphanet. Prévalence des maladies rares: Une enquéte bibliographique. Octobre 2007. Les Cahiers d'orphanet 2007;1-42.

26. Regulation (EC) No $141 / 2000$ of the European Parliament and of the Council of 16 December 1999 on orphan medicinal products: http://eurlex.europa.eu/LexUriServ/site/ es/oj/2000/1_018/1_01820000122es00010005.pdf

27. European Medicines Agency (EMEA). Points to consider on the calculations and reporting of prevalence of a condition for orphan designation. London. 26.Mar.2002 COMP/436/01. http://www.emea.eu.int/pdfs/human/ comp/ 043601.pdf

28. Commission of the European Communities. Commission Staff Working Document on the experience acquired as a result of the application of Regulation (EC) No 141/2000 on orphan medicinal products and account of the public health benefits obtained Document on the basis 01 Article 10 of Regulation (EC). Brusells 2006. http://ec.europa. eu/enterprise/pharmaceuticals/orphanmp/doc/orphan_ en_06-2006.pdf

29. Rare Diseases Task Force. Centres of Reference for rare diseases in Europe: State-of-the-art in 2006 and recommendations of the Rare Diseases Task Force. http://ec.europa.eu/health/ ph_threats(non_com/clocs/contribution_policy.pdf

30. Ministerio de la Salud y de la Protección Social. Plan Nacional Francés para las Enfermedades Raras 2005-2008. Asegurar la equidad para el acceso al diagnóstico, a los tratamientos y a la atención. http://www.eurordis.org/ IMG/pdf/SN_plan_nacional_frances_enfermedades_raras. pdf

31. Pathologies couvertes par les centres de reférence pour une maladie rare ou un groupe de maladies rares labellisés en 2004, 2005 et 2006. http://www.sante.gouv.fr/htm/ dossiers/maladies_rares/maladies_0.htm

32. DG Sanidad y Protección de los Consumidores. Comisión Europea. The Health Programme 2008-2013. http://ec:.europa.eu/health/ph_programme/pgm2008_2013_en.htm

Dr. Luis Carbajal Rodríguez

Encargado de Despacho del Departamento de Medicina Interna. Jefe de la Clínica de Enfermedades por Deposito Lisosomal del Instituto Nacional de Pediatría.

Dra. Juana Inés Navarrete Martínez Servicio de Genética. H.C.S.A.E. PEMEX. 